\title{
Transatlantica
}

Revue d'études américaines. American Studies Journal

\section{Relire The Declining Significance of Race (1978)de William Julius Wilson}

\section{Isabelle Richet}

\section{Q OpenEdition}

1 Journals

\section{Édition électronique}

URL : https://journals.openedition.org/transatlantica/4373

DOI : $10.4000 /$ transatlantica.4373

ISSN : 1765-2766

Éditeur

Association française d'Etudes Américaines (AFEA)

\section{Référence électronique}

Isabelle Richet, « Relire The Declining Significance of Race (1978)de William Julius Wilson »,

Transatlantica [En ligne], 1 | 2009, mis en ligne le 13 juillet 2009, consulté le 20 septembre 2021. URL : http://journals.openedition.org/transatlantica/4373 ; DOI : https://doi.org/10.4000/transatlantica. 4373

Ce document a été généré automatiquement le 20 septembre 2021.

Transatlantica - Revue d'études américaines est mise à disposition selon les termes de la licence Creative Commons Attribution - Pas d'Utilisation Commerciale - Pas de Modification 4.0 International. 


\title{
Relire The Declining Significance of Race (1978)de William Julius Wilson
}

\author{
Isabelle Richet
}

Le moment semble particulièrement bien choisi pour relire, à trente ans de distance, The Declining Significance of Race, l'ouvrage du sociologue afro-américain William Julius Wilson qui, dès sa parution, suscita un virulent débat, en particulier parmi les universitaires noirs. En effet, si l'élection du premier président afro-américain de l'histoire des États-Unis - que Wilson a non seulement soutenu mais conseillé - n'a pas inauguré la société « post-raciale » annoncée un peu vite par certains, elle a néanmoins entraîné chercheurs et politiques à reconsidérer l'importance du facteur racial dans la société étatsunienne. ${ }^{1}$ C'est à une telle réflexion que le sociologue de Harvard appelait ses collègues en 1978, choisissant volontairement un titre provocateur afin d'attirer l'attention sur la thèse centrale de son ouvrage. Pour Wilson, la coexistence entre l'amélioration du statut de certains noirs et la pauvreté persistante des autres était due au fait que la classe jouait désormais un rôle plus important que la race pour déterminer le statut social des afro-américains. Certes, le facteur racial n'avait pas disparu, mais il s'était déplacé de la sphère économique à la sphère sociale et politique et, en conséquence, il proposait de rechercher les causes de la bifurcation évidente des trajectoires sociales au sein de la communauté afro-américaine dans les transformations économiques de l'après-guerre. Il en concluait que les politiques publiques prenant en compte le seul facteur racial - comme l'affirmative action profitaient avant tout à la classe moyenne et ne pouvaient en aucune mesure améliorer le sort du sous-prolétariat noir qui peuplait les ghettos. ${ }^{2}$

2 Il n'était pas le premier à noter la polarisation économique et sociale croissante de la communauté noire. C'était même le point de départ du célèbre rapport de Daniel Patrick Moynihan, rédigé en interne pour l'administration Johnson, puis diffusé publiquement au lendemain des émeutes de Watts d'août 1965 afin d'en suggérer une explication. ${ }^{3}$ Moynihan avait voulu attirer l'attention sur le fait que la visibilité d'une nouvelle classe moyenne noire ne devait pas masquer la situation de la majorité de la population des ghettos qui vivait dans la pauvreté. Pourtant, peu de commentateurs 
relevèrent ce souci réformateur, car le gros du rapport s'attachait à rechercher les causes de cette pauvreté durable dans l'effondrement de la famille noire et l' "enchevêtrement de pathologies" qui en découlait et perpétuait la pauvreté indépendamment de toute action - ou inaction - de la majorité blanche. ${ }^{4}$ Dans le contexte de la montée du féminisme et de l'affirmation du "pouvoir noir», ces analyses rendant le «matriarcat» responsable de la pauvreté des ghettos eurent un effet explosif et influencèrent durablement l'orientation de la recherche sur la communauté afro-américaine. Les chercheurs libéraux, noirs ou blancs, s'attachèrent en effet à réfuter les thèses de Moynihan en démontrant la solidité de la famille noire dans ses diverses configurations et en valorisant la culture de résistance des pauvres du ghetto. ${ }^{5}$ En outre, dans un contexte où le rêve intégrationniste du docteur King cédait le pas à la solidarité de groupe, les différenciations sociales à l'œuvre dans la communauté furent délibérément ignorées et le racisme fut présenté comme le seul obstacle sur la voie du progrès de l'ensemble de la communauté afro-américaine. ${ }^{6}$

C'est dans ce contexte intellectuel que parut The Declining Significance of Race, mais aussi dans le contexte politique et social morose de la fin des années soixante-dix. Non seulement la pauvreté des ghettos s'aggravait sous les coups de la crise et des restructurations économiques que celle-ci entraînait, mais les politiques publiques en faveur de l'égalité raciale semblaient marquer le pas. C'est ce que signalait en particulier l'arrêt Bakke de 1978 dans lequel la Cour suprême rejetait l'usage de quotas dans les admissions à l'université, tout en admettant que le facteur racial pouvait être retenu comme un critère parmi d'autres. ${ }^{7}$ En outre les conservateurs qui fourbissaient alors leurs armes politiques et idéologiques n'hésitaient pas à recycler la carte raciale à travers leur discours moralisateur sur les méfaits du "welfare $\|^{8}$ Contrairement à ce qu'affirmait Wilson dans son ouvrage, aux yeux d'une majorité des dirigeants et des universitaires noirs, le facteur racial restait plus déterminant que jamais pour la trajectoire sociale des Afro-américains. ${ }^{9}$ C'est sur la toile de fond de cette tension entre les analyses avancées par le sociologue et la perception de la situation de la communauté afro-américaine par les politiques et universitaires qui s'exprimaient au nom de celle-ci que se développa la virulente polémique autour de The Declining Significance of Race.

Transatlantica a demandé à un certain nombre d'universitaires travaillant sur la communauté afro-américaine de revenir sur cet ouvrage afin d'éclairer ce débat et d'en cerner les conséquences pour la recherche.

Dans le premier texte de ce dossier, Loïc Wacquant se livre à une triple réflexion à partir de la violente controverse que le livre de Wilson a suscitée parmi les chercheurs afro-américains. Cherchant tout d'abord à comprendre la polémique, il note qu'elle n'a pas été motivée avant tout par l'objet mais par le rapport des chercheurs à l'objet. Si les universitaires noirs ont concentré leur critique sur la description de l'émergence d'une classe moyenne noire alors que Wilson s'intéressait avant tout au sort des habitants défavorisés des ghettos, c'est qu'ils se sont senti visés en tant que personnes privées, membres de cette nouvelle classe moyenne, et non en tant que chercheurs. Le débat ne doit donc pas être vu comme une controverse scientifique mais comme une lutte symbolique pour conserver le monopole de dire la vérité sur la communauté noire. $\mathrm{Au}-$ delà de ce conflit symbolique, Wacquant voit le débat sur l'importance croissante ou décroissante de la race comme un faux problème qui ne peut, posé dans ces termes, trouver une réponse scientifique satisfaisante, car la race - comme la classe - n'est pas 
une catégorie purement objective opérant de façon autonome et indifférenciée dans tous les secteurs de la société. Dans un troisième temps, il appelle à re-conceptualiser l'analyse de la relation entre la race et la classe en abandonnant tout essentialisme pour prendre en compte les luttes à travers lesquelles la race et la classe se construisent à la fois dans l'espace social et théorique. Dans le texte suivant, David Diallo articule sa réflexion autour de la tension entre le "savant " et le "politique ", qui lui paraît la mieux à même de faire sens du débat déclenché par l'ouvrage de Wilson perçu par ses critiques afro-américains plus comme un texte politique que scientifique. Ses critiques opposaient en effet à la démarche sociologique de Wilson, qui portait son attention sur les facteurs extra-raciaux pouvant expliquer la situation économique des Noirs, leur $a$ priori idéologique de tenants de la "black perspective». Exprimant un certain chauvinisme racial, ils voyaient dans The Declining Significance of Race un acte de désolidarisation de la communauté noire. David Diallo rejoint Loïc Wacquant en soulevant la question du rapport à l'objet dans les recherches portant sur les Noirs américains. Dans ses propres travaux sur la culture hip hop, il a pu constater une même myopie analytique qui substitue des récits légitimateurs à une analyse rigoureuse des liens entre cette culture et les pratiques socioculturelles des gangs. Comme au cours du débat autour de l'ouvrage de Wilson, l'idéologie se substitue à l'analyse en violation des règles élémentaires du travail scientifique. Relisant The Declining Significance of Race à partir de son travail sur la famille noire, Hélène Le Dantec-Lowry souligne comment, en introduisant la dimension de classe dans l'étude de l'évolution de la communauté afroaméricaine, William Julius Wilson a permis de sortir des cadres d'analyse essentiellement culturalistes dans lesquels s'enfermait le débat depuis la publication du rapport de Moynihan en 1965. Après avoir passé en revue les travaux des chercheurs conservateurs comme libéraux qui s'étaient emparés des analyses de Moynihan pour finalement produire un même discours stéréotypé sur le ghetto - négatif chez les premiers, positif pour les seconds -, elle montre comment l'approche macrosociologique de Wilson a permis d'appréhender la complexité des schémas de parenté en mettant en évidence la stratification sociale de la communauté noire. Pour Hélène Le Dantec-Lowry, si l'ouvrage de Wilson a permis de renouveler les questionnements, il avait aussi des limites qui ont donné des armes à ses critiques libéraux, mais aussi aux conservateurs qui y ont puisé certains arguments justifiant leurs attaques contre les programmes d'aide sociale. La principale, à ses yeux, est le recours à la notion d'underclass pour désigner un groupe social exclu défini en termes essentiellement culturels - avant tout par un schéma familial «déviant» - qui a favorisé le retour des analyses culturalistes et de la tendance à rendre les exclus responsables de leur situation. Non seulement cette notion encourageait un discours moralisateur sur les pauvres mais elle suggérait un ghetto indifférencié qui ignorait la grande hétérogénéité de l'expérience noire même dans les quartiers défavorisés. En conclusion, elle souligne l'importance d'une articulation plus fine entre les notions de race et de classe qui tienne compte de la réalité différenciée des ghettos, de l'incidence du genre et de la confrontation entre les expériences de classes sociales comparables appartenant à des groupes ethno-raciaux différents. Andrew Diamond, pour sa part, s'interroge d'abord sur l'impact à son avis disproportionné de ce modeste volume et cherche à expliquer pourquoi un auteur qui affiche ses penchants politiques socialdémocrate a apporté tant d'eau au moulin des conservateurs et suscité tant de critiques parmi les chercheurs libéraux ou de gauche. Il trouve une explication dans le contexte de la fin des années soixante-dix. Les démocrates cherchaient à prendre leur distance 
de la politique raciale de leur parti qui avait provoqué tant de divisions en son sein et dans la société et Wilson offrait une certaine légitimité intellectuelle à cette reculade, alors que les Républicains conservateurs, pour leur part, s'emparaient de ses analyses sur le comportement pathologique de l'underclass noire pour remettre en cause les programmes de la guerre contre la pauvreté. Par ailleurs, Diamond souligne comment Wilson est devenu un point de référence négatif motivant un certain nombre d'historiens afro-américains à réorienter leur recherche et, en opposition à sa description désobligeante de l'underclass, à chercher à restituer l'expérience de la classe ouvrière noire dans la perspective de la nouvelle histoire sociale. Les débats autour de la publication de The Declining Significance of Race n'ont pas un intérêt purement historique, tant les questions soulevées par l'ouvrage restent d'actualité. En effet, si la thèse de Wilson à propos de l'importance croissante de la classe dans les trajectoires sociales des afro-américains est désormais largement acceptée, les analyses de l'articulation précise entre race et classe, entre culture et structure sont toujours au centre de la recherche actuelle, comme le montre le plus récent ouvrage de William Julius Wilson lui-même, More Than Just race. Being Black and Poor in the Inner City. ${ }^{10}$

\section{NOTES}

1. Lawrence D. Bobo, Camille Z. Charles, "Race in the American Mind: From the Moynihan Report to the Obama Candidacy," Annals of the American Economy of Political Science, vol. 621, 1, janvier 2009, 243-259; Richard Alba, Blurring the Color Line: The New Chance for a More Integrated America, Cambridge, Harvard University Press, 2009.

2. William Julius Wilson, The Declining Significance of Race, Chicago, University of Chicago Press, 1978.

3. Carl Gershman, «A Matter of Class », New York Times Magazine, 5 octobre 1980.

4. Le rapport intitulé The Negro Family. The Case for National Action est disponible en ligne : http:// www.dol.gov/oasam/programs/history/webid-meynihan.htm

5. Douglas S. Massey \& Robert J. Sampson, "Moynihan Redux: Legacies and Lessons", Annals of the American Academy of Political and Social Science, vol. 621, 1, janvier 2009, 9, 12.

6. William Julius Wilson, "The Moynihan Report and Research on the Black Community”, Annals of the American Academy of Political and Social Science, cit., 36-37.

7. Regents of the University of California v. Bakke, 438 U.S. 265 (1978)

8. Cf. Thomas Byrne Edsall \& Mary D. Edsall, Chain Reaction: The Impact of Race, Rights and Taxes on American Politics, New York, W.W. Norton, 1992; Dan T. Carter, From George Wallace to Newt Gingrich: Race in the Conservative Counterrevolution, Baton Rouge, LA, Louisiana University Press, 1999.

9. Cf. Carl Gershman, «A Matter of Class », New York Times Magazine, 5 octobre 1980.

10. William Julius Wilson, More Than Just Race. Being Black and Poor in the Inner City, New York, W.W. Norton, 2009. Voir aussi, parmi les recherches les plus récentes, Michèle Lamont et Mario Small, "How Culture Matters for the Understanding of Poverty: Enriching our Understanding", in Ann Chih Lin, David D. Harris (dir.) The Colors of Poverty: Why Racial and Ethnic Disparities Exist, New York, Russell Sage, 2008. On lira aussi avec intérêt la livraison de janvier 2009 de Annals of the American Academy of Political and Social Science, sous la direction de Douglas S. Massey et Robert J. 
Sampson, entièrement consacrée à une discussion du rapport Moynihan à quatre décennies de distance.

\section{AUTEUR}

ISABELLE RICHET

Université Paris-Diderot (LARCA) 